\title{
Novel functions of LHX2 and PAX6 in the developing telencephalon revealed upon combined loss of both genes
}

\author{
Geeta Godbole, Achira Roy, Ashwin S. Shetty and Shubha Tole* (D)
}

\begin{abstract}
Patterning of the telencephalic neuroepithelium is a tightly regulated process controlled by transcription factors and signalling molecules. The cortical primordium is flanked by two signalling centres, the hem medially, and the antihem laterally. The hem induces the formation of the hippocampus in adjacent neuroepithelium. Therefore, the position of the hem defines the position of the hippocampus in the brain. The antihem is positioned at the boundary between the dorsal and ventral telencephalon and proposed to provide patterning cues during development. LIM-homeodomain (LIM-HD) transcription factor LHX2 suppresses both hem and antihem fate in the cortical neuroepithelium. Upon loss of $L h \times 2$, medial cortical neuroepithelium is transformed into hem, whereas lateral cortical neuroepithelium is transformed into antihem. Here, we show that transcription factor PAX6, known to regulate patterning of the lateral telencephalon, restricts this tissue from transforming into hem upon loss of Lhx2. When Lhx2 and Pax6 are both deleted, the cortical hem expands to occupy almost the complete extent of the cortical primordium, indicating that both factors act to suppress hem fate in the lateral telencephalon. Furthermore, the shift in the pallial-subpallial boundary and absence of the antihem, observed in the Pax6 mutant, are both restored in the $L h \times 2$; Pax6 double mutant.

Together, these results not only reveal a novel function for LHX2 in regulating dorsoventral patterning in the telencephalon, but also identify PAX6 as a fundamental regulator of where the hem can form, and therefore implicate this molecule as a determinant of hippocampal positioning.
\end{abstract}

Keywords: Organizer, Dorsoventral, Patterning, Craniofacial, Mediolateral, Hem

\section{Introduction}

In development, tissues are patterned by the action of morphogens secreted by signaling centers known as "organizers." The WNT- and BMP- secreting cortical hem in the telencephalon is one such medial signaling center $[1,2]$. At the lateral edge of the cortical neuroepithelium, the EGF-expressing antihem defines the boundary between the dorsal and ventral telencephalon [3].

Previously, we demonstrated that the hem is capable of organizer function, by creating embryos with multiple hems in the telencephalon, and showing that each ectopic hem induces the formation of an ectopic hippocampus [4]. The factors that act to restrict the hem to its position at the medial edge of the cortical neuroepithelium are therefore critical determinants of where the hippocampus

\footnotetext{
* Correspondence: shubhatole@gmail.com

Department of Biological Sciences, Tata Institute of Fundamental Research, Mumbai, India
}

will form in the brain. Transcription factor LHX2 is a critical suppressor of the cortical hem. In the $L h \times 2$ null mutant, the cortical primordium cannot retain its identity, but is transformed into two alternate fates: medial cortical neuroepithelium is transformed into hem, whereas lateral cortical neuroepithelium is transformed into antihem [4, 5]. In mosaic experiments, $L h x 2$ null clones in the medial telencephalon display hem fate, and those in the lateral telencephalon display antihem fate, indicating a cell-autonomous requirement for LHX2 in cortical neuroepithelial cells [4]. Why loss of $L h x 2$ leads to two disparate fate transformations in the dorsal telencephalon is unclear.

Paired domain containing homeobox transcription factor PAX6 is required for antihem fate, which is lost in Pax6 null mutants [3]. Pax6 is expressed in a lateral(high)medial(low) gradient, opposite to that of $\operatorname{Lhx} 2[6,7]$. We hypothesized that the presence of PAX6 may restrict the medio-lateral extent of the hem in $L h x 2$ 
mutants. Supporting this hypothesis, in the mosaic experiments performed in Mangale et al. (2008), PAX6 was lost in medial $L h x 2$ null patches that formed ectopic hems, but not in lateral $L h x 2$ null patches that formed antihem. Here, we examined medio-lateral patterning in embryos lacking both $L h x 2$ and Pax6, using null mutants as well as conditional knockout strategies. We find that the hem indeed expands to encompass almost the entire dorsal telencephalon when both $L h x 2$ and Pax6 are lost. Concomitantly, the loss of both factors appears to partially restore dorsoventral patterning that is disrupted in the Pax6 single mutant [8]. Thus, an examination of the Lhx2;Pax6 double mutant uncovers novel functions for both transcription factors, not seen upon loss of either factor alone. Our results show LHX2 to be a regulator of dorsoventral patterning in the telencephalon. PAX6 emerges as a key player in controlling the medial position of the hippocampus, because it prevents the hippocampal organizer, the hem, from being formed at lateral positions.

\section{Results and discussion}

We used an $L h x 2$ null mutant line generated by targeted gene disruption [9] and the $P a x 6^{\text {sey/sey }}$ line which has a point mutation in the Pax6 gene creating a null allele [10] to generate the $L h x 2^{-/-} ; \mathrm{Pax}^{\text {sey/sey }}$ double null mouse mutant. This mutant suffers from gross craniofacial abnormalities including an absence of external eyes, similar to the individual null phenotypes for $\operatorname{Lh} x 2^{-/-}$and $\mathrm{Pax}^{\mathrm{sey} / \mathrm{sey}}$ embryos, and also lacks external nostrils similar to $P a x 6^{\text {sey/sey }}[9,11]$. A unique feature in the double null is the presence of cleft-lip/palate, indicating a combinatorial role for LHX2 and PAX6 in craniofacial development (Additional file 1: Figure S1). To our knowledge, this is the first characterization of an Lhx2;Pax6 double null mutant.

\section{PAX6 and LHX2 together restrict medial cortical fate to the medial telencephalon}

We analyzed $L h x 2^{-1-} ; \mathrm{Pax}^{\text {sey/sey }}$ double mutants at embryonic day (E) 12.5 with markers of medial telencephalic structures and compared the results with the expression seen in the respective single null mutants. We examined medial cortical marker Wnt8b which identifies the hem and the hippocampal primordium, and septum marker Fgf17. As expected, Wnt8b expression marks the medial telencephalic primordium in the $\mathrm{Pax}^{\mathrm{sey} / \mathrm{sey}}$ mutant. In the $L h \times 2^{-/-}$mutant, Wht8b is expressed in the expanded hem, sparing some lateral tissue. In contrast, loss of both genes results in the entire dorsal telencephalon expressing medial markers, apparently sparing no lateral cortical neuroepithelium (Fig. 1). However, the double null mutant brains were fragile and the morphology made it difficult to analyze this apparent lateral-to-medial transition properly. Furthermore, the individual null mutants of $L h x 2$ and Pax6 are embryonic lethal $[9,10]$, and both genes lie on chromosome 2, which makes it difficult to obtain homozygous $L h \times 2^{-/-} ; \operatorname{Pax} 6^{\text {sey/sey }}$ double null mutants

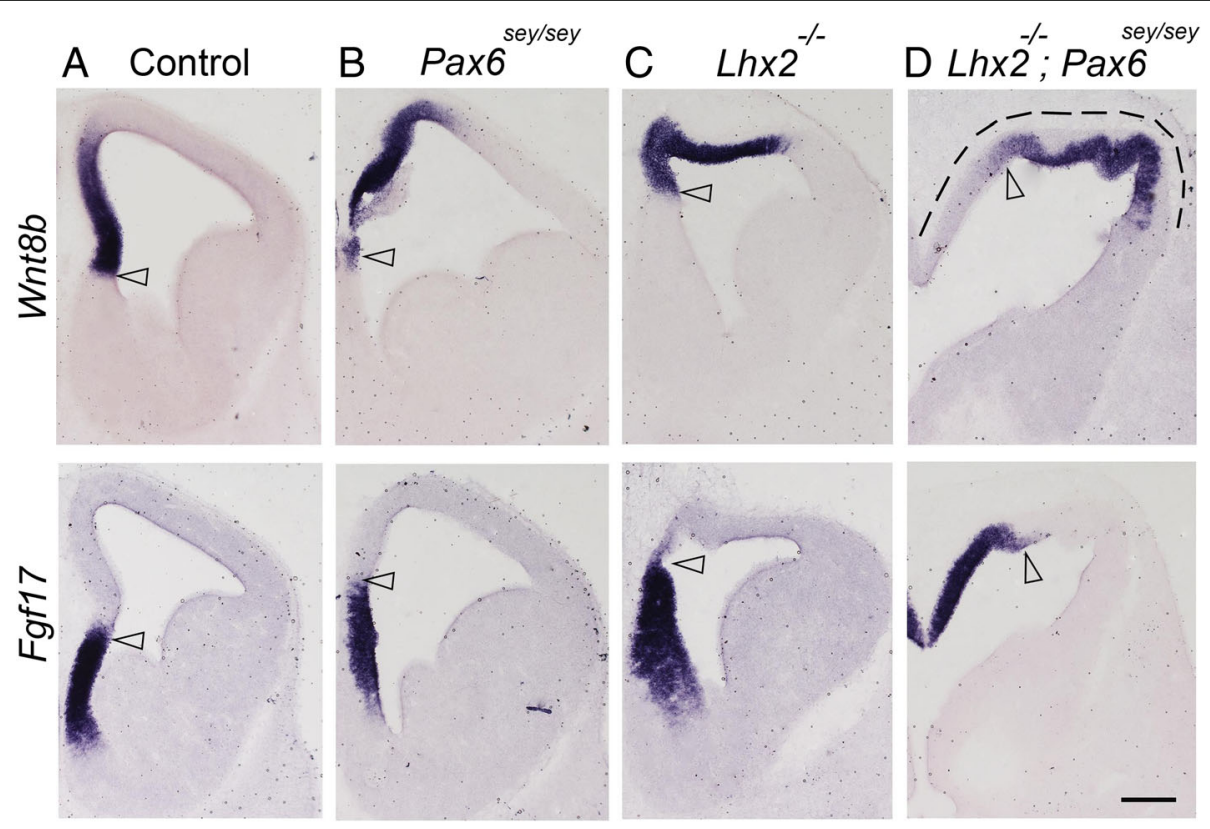

Fig. 1 Medial fates are expanded laterally upon loss of both LHX2 and PAX6. a-d E12.5 coronal sections of Control (a), Pax6 $6^{\text {sey/sey }}(\mathbf{b})$, LhX2 $2^{-/-}(\mathbf{c})$, and $L h \times 2^{-1-} ; P \times 6^{\text {sey/sey }}$ (d) were examined for the expression of medial cortical marker Wnt8b and septum marker Fgf17, which identify adjacent, non-overlapping regions of the medial telencephalon (open arrowheads mark the boundary between the two markers). In the double mutant, these medial markers together encompass the entire dorsal telencephalon (dashed line, d). Scale bar is $200 \mu m$ 
from heterozygous crosses. Therefore, we generated Lh $x 2^{\text {lox } / l o x} ; \operatorname{Pax}^{\text {lox } / l o x}$ double conditional mutants and crossed them with a ubiquituously expressed tamoxifen inducible CreER line.

\section{Lhx2 and Pax6 double conditional mutants phenocopy the patterning defects in the corresponding double null mutants}

We tested whether administration of tamoxifen at E8.5 to $C r e E R ; L h x 2^{l o x / l o x} ; P a x 6^{l o x / l o x}$ animals recapitulates the results we obtained from the respective single and double null mutants by E12.5. For all experiments using $L h x 2$ and Pax6 single or double conditional mutant embryos, we examined the expression of the respective floxed exon(s) in each embryo in serial sections, and only used embryos which displayed extensive recombination for analysis. One example of an $L h x 2^{l o x / l o x} ; \operatorname{Pax} 6^{l o x / l o x}$ double conditional mutant brain is shown in Additional file 2: Figure S2. Loss of Pax6 is not reported to affect patterning of the dorsomedial telencephalon [12] and indeed, the extent of Wnt $8 b$ expressing neuroepithelium appears comparable in Pax $6^{\text {lox/lox }}$ and control embryos (Fig. 2a, b). In $L h x 2^{\text {lox } / l o x}$ embryos, Wnt8b expression appears to spare some lateral telencephalic tissue, which is presumably the antihem [4]. In the $L h x 2^{\text {lox/lox }}$; $P a x 6^{\text {lox/lox }}$ double conditional mutant brains, Wnt8b expression appears to extend upto the pallial-subpallial boundary, suggesting that lateral cortical fates are missing (Fig. 2d). We therefore proceeded with these double conditional mutants for further analysis of the nature of this apparent lateral-to-medial transformation upon loss of both $L h x 2$ and Pax6. Furthermore, because the boundary between the dorsal and ventral telencephalon is difficult to identify accurately in the double mutant by morphology alone, we used $D b x 1$ expression to identify it in subsequent experiments. $D b x 1$, a marker of the antihem $[13,14]$ is expressed in a subpopulation of scattered cells in the ventricular zone of this structure [15].

\section{The hem expands to a greater extent upon loss of both $L h \times 2$ and Pax6 than upon loss of $L h \times 2$ alone}

Since loss of Pax6 alone did not expand medial fates (Fig. 2), we focused only on $L h x 2$ single and $L h x 2$;Pax6 double conditional mutants for further analysis. We administered tamoxifen at E8.5 to CreER; $L h x 2^{\text {lox } / l o x}$ single conditional mutant mice and to CreER; Lhx $2^{\text {lox } / \text { lox }} ; \operatorname{Pax} 6^{\text {lox/lox }}$ double conditional mutant mice and harvested the embryos at E12.5. We used a set of three markers, Wnt2b, Wnt3a, and Lmxla, to examine the extent of the hem in these mice. All three markers reveal a greater expansion of the hem upon loss of both $L h x 2$ and Pax6 compared with that seen upon loss of $L h x 2$ alone (Fig. 3). We quantified the extent of hem expansion by examining Wnt3a expression in control, Lhx2 single conditional mutants, and Lhx2;Pax6 double

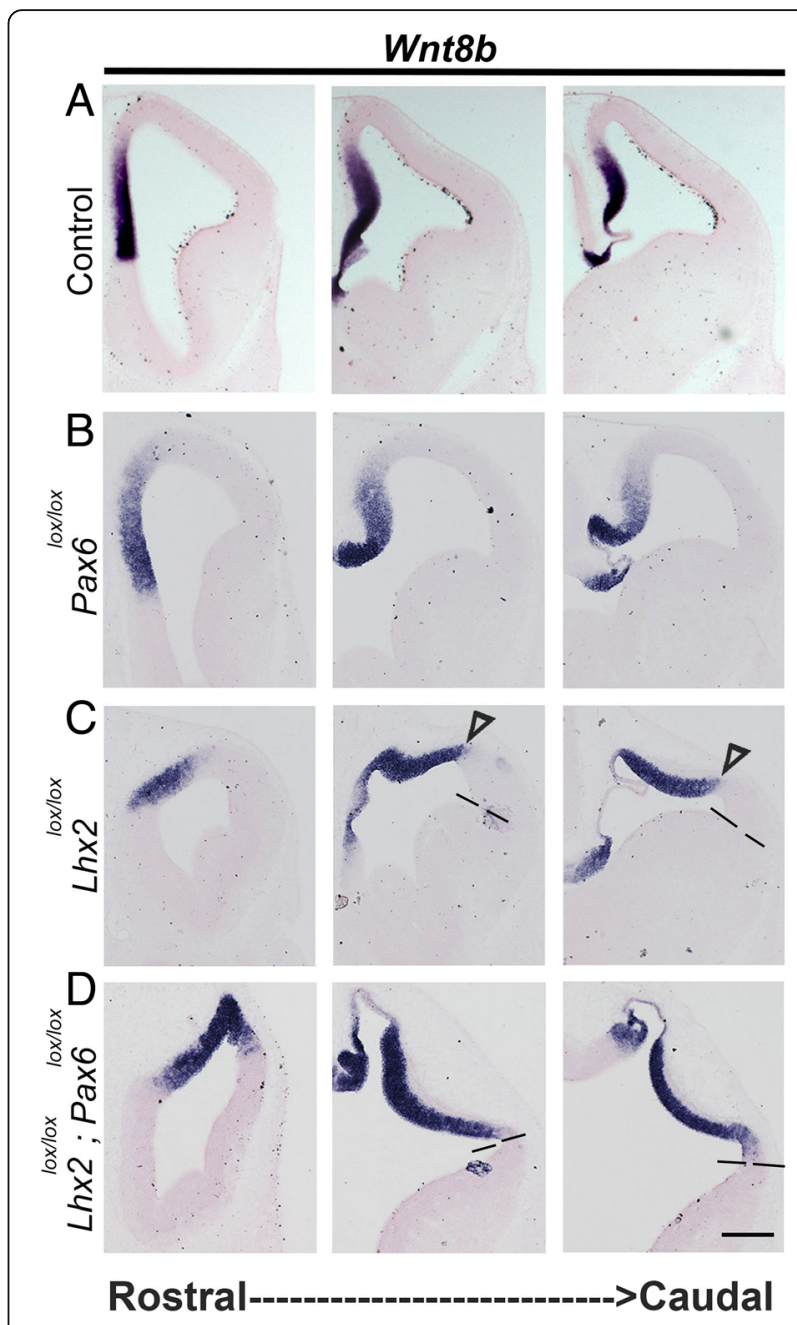

Fig. 2 Conditional deletion of $L h \times 2$ and Pax6 from E8.5 recapitulates the lateralization of medial fates seen in the double null mutants. a-d Tamoxifen was administered at E8.5 to Control (a), CreER:Pax6 ${ }^{10 \times 10 \times}(\mathbf{b})$,

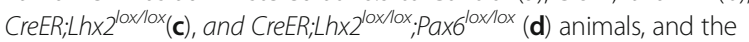
embryos were harvested at E12.5. Coronal sections were examined for Wnt8b expression at rostral, mid, and caudal levels of sectioning. Wnt8b expression is restricted to the medial cortical neuroepithelium in control and Pax $6^{\text {lox/10x }}$ brains $(\mathbf{a}, \mathbf{b})$. In $L h \times 2^{\text {lox/lox }}$ brains, a small region of lateral neuroepithelium remains Wnt8b-negative (region between open arrowhead and dashed line, c). In the double conditional mutants, Wnt8b expression extends almost upto the pallial-subpallial boundary (dashed lines, $\mathbf{c}$, d). Scale bar is $200 \mu \mathrm{m}$

conditional mutants (Fig. 3a-d). We calculated the length of the hem as a fraction of the total dorsal telencephalic neuroepithelium measured up to the ventral extent of $D b x 1$ expression in an adjacent section $(D b x 1$ sections are shown in Additional file 3: Figure S3). We scored rostral, medial, and caudal levels separately and found that there was no significant rostro-caudal difference in the fractional hem length in a given mutant. As is apparent from the images, the hem is longer in $L h \times 2$ single conditional mutants than in control brains. The key comparison of the $L h x 2$ single with the Lhx2;Pax6 double conditional mutants reveals that the 


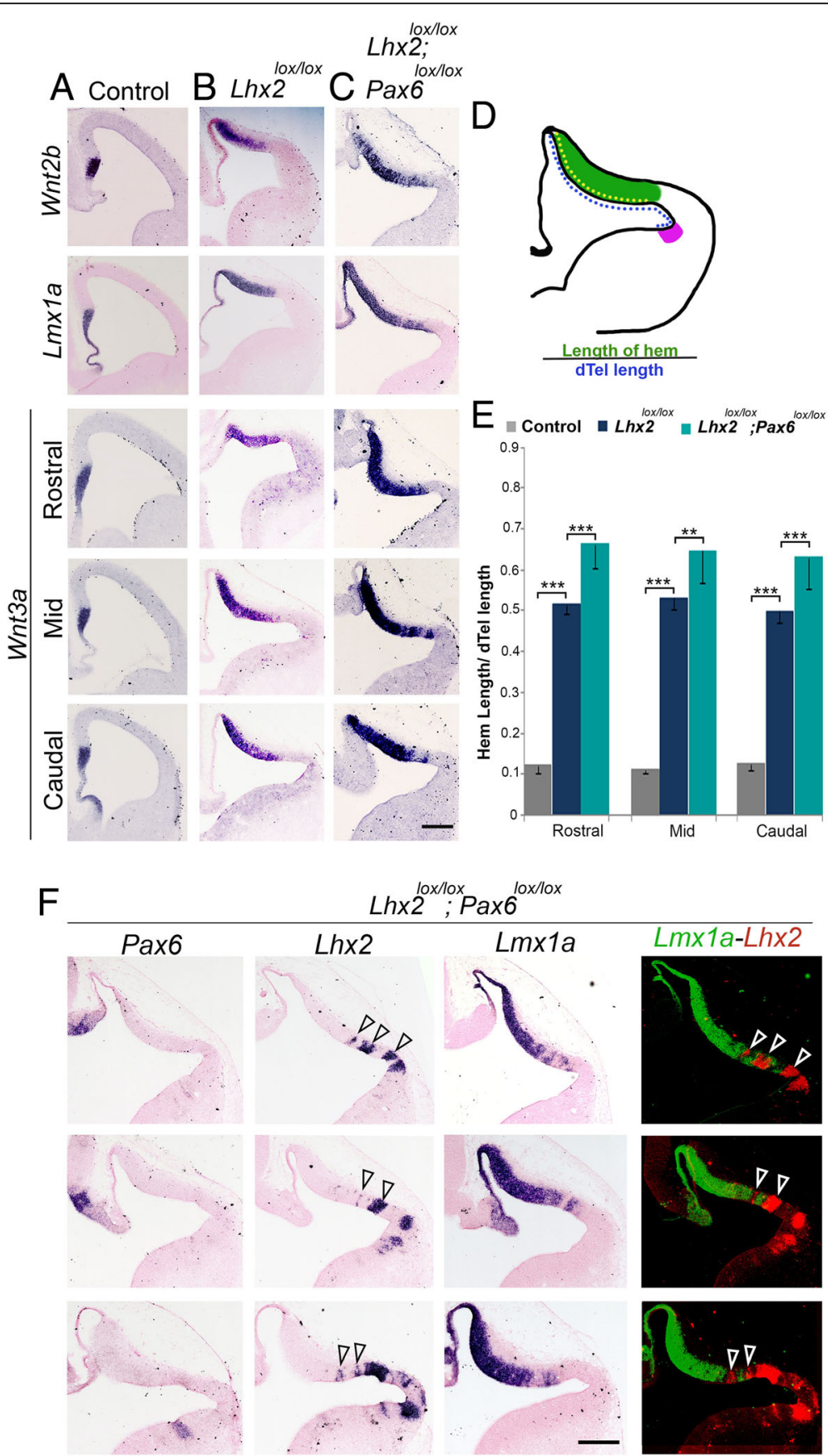

Fig. 3 PAX6 restricts the lateral extent to which the hem can expand when LHX2 is lost. (a-c; $\mathbf{f}$ ) Tamoxifen was administered at E8.5 to Control

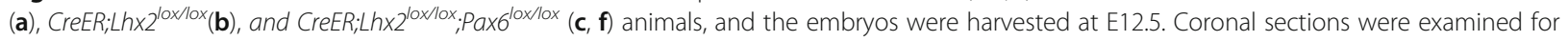
the expression of hem markers Wnt2b and Lmx1a. Wnt3a was examined at rostral, mid, and caudal levels of sectioning. In each condition, the length of the hem was scored as a fraction of the total length of the dorsal telencephalic neuroepithelium measured up to the ventral extent of Dbx1 expression in an adjacent section (shown in Additional file 3: Figure S3). $\mathbf{d}$ cartoon illustrating the length measurement (yellow dotted line) of the hem (green). Blue dotted line indicates the length measurement of the dorsal telencephalic neuroepithelium measured upto the ventral extent of antihem (pink). e Quantification of the fractional length of the hem in control Lhx2 single conditional mutants, and Lhx2;Pax6 double conditional mutants, at rostral, mid, and caudal levels of sectioning. For each level, the single mutant hem was significantly longer than that in the control, and the double mutant hem was significantly longer than that in the single mutant or the control $\left({ }^{* *} p<0.005,{ }^{* * *} p<0.0005\right)$. f Adjacent sections from three different double conditional mutant embryos in which $L$ hx 2 was incompletely recombined were examined. In each case, when Pax6 is completely removed, but $L h \times 2$ is present in patches (open arrowheads), the hem is suppressed in $L h \times 2$ expressing regions. A false-color overlay shows that hem (green) is not seen in patches that show Lhx2 expression (red; open arrowheads). Scale bar is $200 \mu \mathrm{m}$ 
fractional length of the hem is significantly greater when both factors are lost compared with the loss of $L h \times 2$ alone (Fig. 3e). This indicates that PAX6 functions to restrict the lateral expansion of the hem in an $L h x 2$ mutant background.

\section{LHX2 suppresses hem fate at the lateral extreme of the dorsal telencephalon in a Pax6 mutant background}

Tamoxifen-driven Cre recombination is sometimes incomplete and results in patches of tissue that escape recombination. When we examined the lateral telencephalon of embryos that displayed complete recombination of Pax6 but had some unrecombined patches of $L h \times 2$, it was apparent that hem only formed where both genes were lacking (Fig. 3f). That is, the presence of $L h x 2$ in a Pax6 mutant background was enough to suppress hem fate. This demonstrates that LHX2 is a suppressor of hem fate both medially and laterally, the latter function being revealed only in a Pax6 mutant background.
In summary, these results demonstrate that the extreme medial fate of the cortical hem can be generated at extreme lateral positions, and that LHX2 and PAX6 each suppresses hem fate. These roles of LHX2 and PAX6 are revealed only upon analysis of the double mutants, and could not have been predicted from examining either single mutant alone.

\section{Absence of Lhx2 and Pax6 partially restores the pallial-subpallial boundary/antihem that is disrupted upon loss of Pax6 alone}

Finally, we examined the pallial-subpallial boundary itself, since PAX6 is known to be a regulator of the position of this border $[12,16]$. We administered tamoxifen at E8.5 to conditionally remove $L h x 2$ alone, Pax 6 alone, or both, and harvested the embryos at E12.5. The expression of both Neurog2 and Gsx2 stops at the pallial-subpallial boundary, with Neurog 2 being expressed in the pallial side and Gs $x 2$ in the subpallial side (Fig. 4, [8]). Loss of $L h x 2$ does not appear to alter these expression boundaries, though it

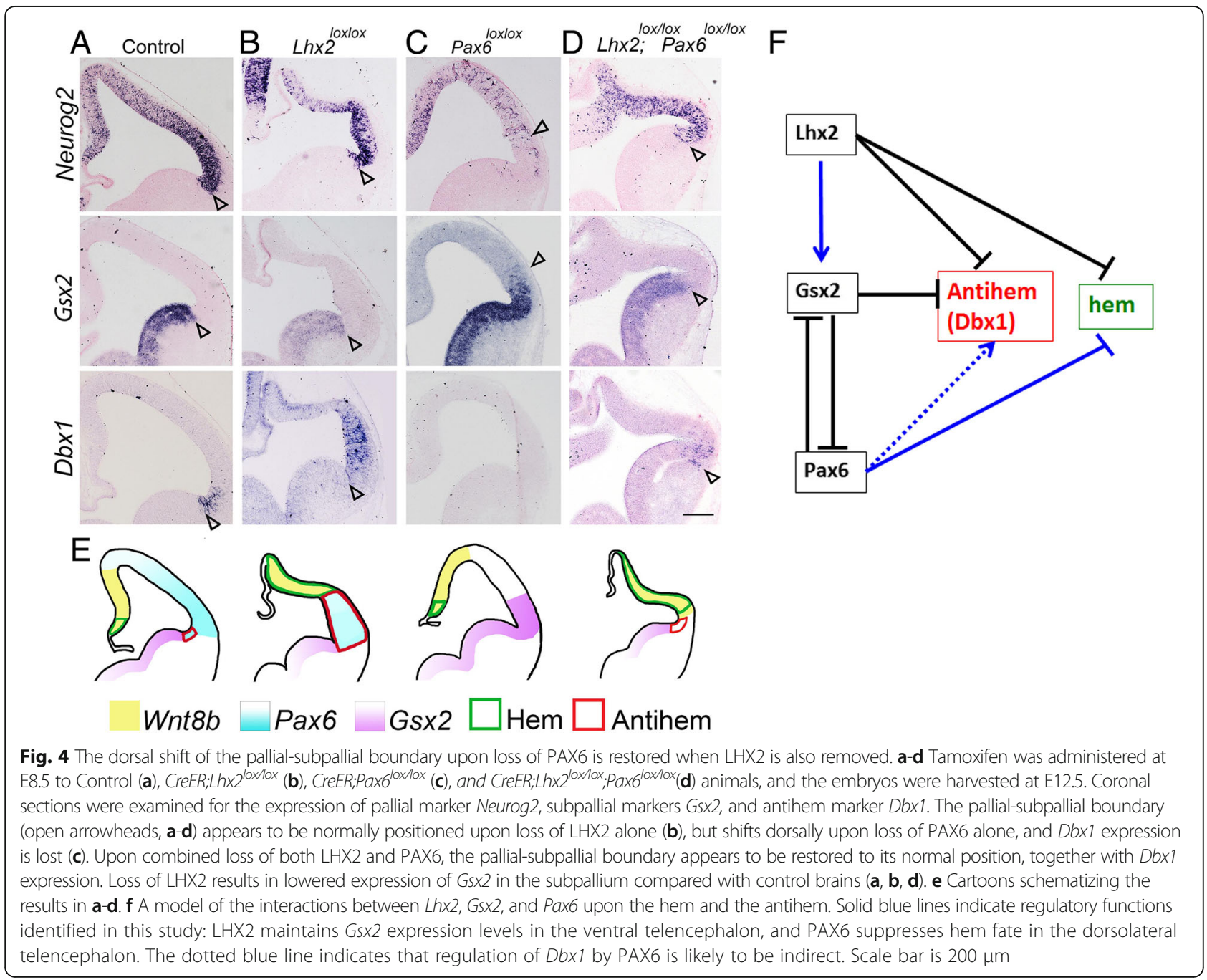


does appear to cause an overall reduction in the level of Gsx2 expression in the subpallium (Fig. 4). In the absence of Pax6, the pallial-subpallial boundary is known to shift dorsally, with Gsx 2 expression seen in the lateral region of the pallium where Neurog2 expression recedes (Fig. 4; $[8,16])$. This is thought to be due to cross-repression between PAX6 and GSX2 [8, 13]. However, since LHX2 appears to be required for normal Gs $x 2$ expression, when $L h x 2$ and Pax6 are both lost, the expanded Gsx2 expression in the lateral neuroepithelium is no longer seen, but instead, Neurog 2 is expressed there. Thus, despite the absence of Pax6, the additional loss of $L h \times 2$ appears to restore the pallial-subpallial boundary.

This regulation of the pallial-subpallial boundary has an intriguing effect on $D b x 1$ expression. GSX2 is known to suppress $D b x 1$ and prevent it from being expressed in the subpallium, and consistent with this, $D b x 1$ expands into the subpallium in Gs $x 2$ mutants [15]. Upon loss of Pax6, Gsx2 expression occupies the region of the antihem and suppresses $D b x 1$ expression there [15]. Thus, the loss of $D b x 1$ expression in the antihem of Pax6 mutants is likely to be indirect, via GSX2. LHX2 also suppresses $D b x 1$ expression [4] and promotes Gsx2 expression (the current study). The expansion of Gs $x 2$ into pallial territory upon loss of Pax6 is reversed upon the additional loss of $L h x 2$. The reappearance of $D b x 1$ in its apparently normal antihem location in the $L h x 2^{l o x / l o x} ; \mathrm{Pax}^{\text {lox/lox }}$ double conditional mutant brain may be because both its suppressors- LHX2 or GSX2- are absent in this location.

Therefore, though $D b x 1$ expression is undetectable in the Pax6 single mutant, it is clear from its reappearance (albeit in an ectopic location) in the $P a x 6^{\text {sey/sey }}$; Gs $x 2^{-/-}$double null mutant [15], and in an apparently normal location in the $L h x 2^{\text {lox/lox }} ; \operatorname{Pax}^{\text {lox } / l o x}$ double conditional mutant (this study), that $D b x 1$ does not require PAX6, per se, for its expression. Like $D b x 1$, sFrp2 expression is also lost in the $P a x 6^{\text {sey/sey }}$ brain [15]. However, in contrast to $D b x 1$, we find that $s F r p 2$ does not reappear in the $L h x 2^{\text {lox/lox}} ; \mathrm{Pax}^{\text {lox/lox }}$ double mutant (Additional file 4: Figure S4). Since loss of $L h \times 2$ alone does not cause loss of $s F r p 2$ expression [17], this suggests that $s F r p 2$ critically requires PAX6 for its expression. This indicates that different molecular features of the antihem are regulated by distinct combinations of factors, underscoring the heterogeneity of this structure.

\section{Conclusion}

This study uncovers new roles for transcription factors LHX2 and PAX6 in patterning neuroepithelial domains in the telencephalon. LHX2 was known to suppress hem fate in the medial component of the dorsal telencephalon, but we find that PAX6 and LHX2 together suppress hem fate in the lateral component of the dorsal telencephalon as well. We discover that hem fate can indeed arise at the lateral extreme of the dorsal telencephalon, almost upto the pallial-subpallial boundary, if both PAX6 and LHX2 are removed. Therefore, this demonstrates that PAX6 plays a role in suppressing hem fate. Finally, we show that the LHX2 plays a role in regulating the pallial-subpallial boundary. This is surprising since LHX2 is expressed in a gradient in the entire telencephalic ventricular zone, and does not display an expression boundary in the lateral telencephalon. This role for LHX2 is partly due to its regulation of Gsx2 in the ventral telencephalon, which is a novel genetic interaction between these two developmental control molecules.

Our findings are summarized in the model in Fig. 4, which illustrates how LHX2 and PAX6 interact to regulate the positioning of the hem, the expression of the antihem marker $D b x 1$ and also where the pallialsubpallial boundary is formed. These roles for LHX2 and PAX6 are revealed only upon analysis of the double mutants, and could not have been predicted from examining either single mutant alone, thus highlighting the importance of these "classical" epistasis studies in the developing brain. More broadly, an approach that examines the functions of specific factors in the context of different mutant backgrounds may reveal unexpected spatiotemporal interactions between genes and could raise new hypotheses for the control of developmental phenomena. This may be particularly useful in understanding disorders such as autism or other neurodevelopmental diseases in which the severity of the phenotype might depend on the combinations and levels of proteins that may not appear likely to interact with each other based on standard single gene mutation studies.

\section{Material and methods \\ Mice}

All animal protocols were approved by the Institutional Animal Ethics Committee (Tata Institute of Fundamental Research, Mumbai, India) according to regulations devised by the Committee for the Purpose of Control and Supervision of Experiments on Animals (CPCSEA), India. The tamoxifen-inducible CreERT2 line (strain name: B6; 129-Gt(ROSA)26Sortm1(Cre/ERT)Nat/J; stock number: 004847) was obtained from the Jackson Laboratory. The $L h \times 2^{-/-}$line used in this study was a kind gift from Forbes D. Porter, NIH, USA [9]. The $L h \times 2^{l o x / l o x}$ line used in this study was a kind gift from Edwin Monuki, University of California, Irvine, USA [4]. The Pax $6^{\text {lox/lox }}$ line was a kind gift from David Price, University of Edinburgh [18] and the $\mathrm{Pax}^{\mathrm{Sey} / \mathrm{sey}}$ line was a kind gift from Anastassia Stoykova, MPI Goettingen, Germany [12].

Noon of the day of vaginal plug was designated as embryonic day 0.5 (E0.5). Tamoxifen (Sigma, MO, USA) was administered to the pregnant dams at different time points as mentioned in the text and embryos were 
harvested at E12.5. Control embryos were littermates with one wild-type copy of the relevant gene. The tamoxifen dosage administered was $75 \mu \mathrm{g} / \mathrm{g}$ body weight.

\section{Sample preparation and in situ hybridization}

Freshly harvested brains were fixed in $4 \%$ paraformaldehyde (PFA) (Sigma, MO, USA) overnight and equilibrated in $15 \%$ sucrose followed by $30 \%$ sucrose (SRL Chem, Mumbai, India). The brains were sectioned at $16 \mu \mathrm{m}$ sections using a freezing microtome and mounted on Superfrost Plus slides (Electron Microscopy Sciences, Hatfield, PA, USA). Sections were post-fixed in 4\% PFA, washed in Phosphate buffered Saline (PBS), and treated with proteinase $\mathrm{K}$ (Sigma, MO, USA) $(1 \mu \mathrm{g} / \mathrm{ml})$ at $37^{\circ} \mathrm{C}$ for 10 mins. One more round of post-fixing and PBS washes was performed, and, the sections were incubated in hybridization buffer (5X SSC, 50\% formamide, 1\% SDS) containing different antisense RNA probes at $70^{\circ} \mathrm{C}$ overnight. Probes were prepared using a kit from Roche per the manufacturer's instructions (Roche, Mannheim, Germany). The next day, after washes with solution X (2X Saline sodium citrate SSC, 50\%formamide, $1 \%$ SDS) at $70^{\circ} \mathrm{C}$, followed by stringent washes with $2 \mathrm{X}$ SSC and $0.2 \mathrm{XSC}$ at room temperature, the sections were washed with TBST $(10 \mathrm{mM}$ Tris- $\mathrm{HCl}, \mathrm{pH} 7.5$, and $150 \mathrm{mM} \mathrm{NaCl}$, $0.1 \% \mathrm{KCl}, 0.5 \%$ Tween-20). The slides were then incubated with alkaline phosphatase conjugated anti-Digoxigenin Fab fragments (1:5000, Roche, IN, USA) for $16 \mathrm{~h}$ at $4^{\circ} \mathrm{C}$. The slides were then washed four times with TBST and then with developing buffer NTMT (100 mM NaCl, $100 \mathrm{mM}$ Tris, pH 9.5, $50 \mathrm{mM} \mathrm{MgCl}, 1 \%$ Tween-20). The color reaction was performed using Nitro-blue tetrazolium chloride and 5-bromo-4-chloro-3-indolyl-phosphate (NBT-BCIP, Roche, IN, USA) as per manufacturer's instructions. The incubation was performed for 10-40 h, and terminated when the color reaction had developed satisfactorily, as assessed by the intensity of signal and low background. The reaction was stopped in Tris-EDTA (10 mM Tris- $\mathrm{HCl}(\mathrm{pH} 7.5)$, $10 \mathrm{mM}$ EDTA ( $\mathrm{pH}$ 8.0) and fixed 3.7\% formaldehyde (diluted in PBS from a 37\% stock, Sigma for $1 \mathrm{~h}$ at RT. Finally, the slides were washed in PBS, dried and mounted in DPX mountant (S.D Fine Chem, Mumbai, India).

To identify unrecombined cells, an RNA probe against Lhx 2 exon $2 / 3$ and Pax6 exon 5/5a/6 were made by PCR followed by in vitro transcription (Roche, Mannheim, Germany) as per the manufacturer's instructions. The primers used were as follows;

Lhx2 exon 2/3 forward: 5' CGCGGATCCACCATGCC GTCCATCAGC 3';

Lhx2 exon 2/3 reverse: 5' GGCGTTGTAAGCTGCCAG 3';

Pax6 exon5/5a/6 forward: 5' TCACAGCGGAGTGAA TCAGCTT 3';

Pax6 exon 5/5a/6 reverse: 5' CACTGGGTATGTTATC GTTG 3'.
For each embryo, the extent of recombination was examined by in situ hybridization for the recombined exon in one series of sections. All mutant combinations were examined in at least 4 embryos of the relevant genotype.

\section{Image acquisition and analysis}

Bright field images were acquired by Zeiss microscope Axioskop 2plus using a Nikon DS-fi2 camera. Length measurements were obtained using ImageJ software from eight hemispheres ( $n=8$, from 4 brains) of each genotype: control, single $L h \times 2$ conditional mutants and $L h \times 2 ; P a x 6$ double conditional mutants. The hem length was measured as the length of neuroepithelium expressing Wnt3a, and dorsal telencephalic neuroepithelium length was measured from the medial edge of the hem up to the ventral extent of $D b x 1$ expression in an adjacent section (shown in Additional file 3: Figure S3). For each hemisphere, the ratio of the hem length to the total dorsal telencephalic neuroepithelium length was determined at rostral, medial, and caudal levels of sectioning. The mean and standard deviation (SD) were calculated, and one-way ANOVA followed by the Tukey's post-hoc test was used to determine significance. Statistical analyses were performed using Graph Pad Prism 5. Error bars in the figure indicate standard deviation $(\mathrm{SD})$.

\section{Additional file}

\begin{abstract}
Additional file 1: Figure S1. Constitutive loss of both $L h \times 2$ and Pax6 results in severe cranio-facial defects. $(A, B)$ lateral views and $(C, D)$ dorsal views of E12.5 heads from control $(A, C)$ and $L h \times 2^{-1-}$;Pax $6^{\text {sey/sey }}$ double mutant $(B, D)$ embryos. A control embryo shows the presence of optic cups (black arrowhead) and nostrils (white arrowhead), while the double mutant lacks eyes (black asterisk) and displays two protruding structures in place of the nasal mass (open arrowheads), suggestive of an open or cleft palate (JPEG $965 \mathrm{~kb}$ )

Additional file 2: Figure S2. Tamoxifen was administered at E8.5 to CreER; Lhx $2^{\text {lox/10x }}$ :Pax6 ${ }^{\text {lox/10x }}$ animals and the embryos were harvested at E12.5. Near-complete recombination of Pax 6 and $L h \times 2$ is seen in a rostrocaudal series of sections adjacent to those examined for Wnt8b expression. (JPEG 807 kb)

Additional file 3: Figure S3. This figure shows $D b \times 1$ expression in adjacent sections to the images shown in Fig. 3. These images were used for measurements of the hem and the dorsal telencephalic neuroepithelium, taken upto the ventral extent of Dbx 1 in adjacent sections in CreERT2; Lhx $2^{\text {lox/lox }}$ (dashed lines, $\left.\mathrm{B}, \mathrm{D}, \mathrm{F}\right)$ and CreER;L $\mathrm{L} \times 2^{\text {lox/lox}} ; \mathrm{Pax} 6^{\text {lox/lox }}(\mathrm{H}, \mathrm{J}, \mathrm{L})$ embryos. Dbx1 expression in the control brain is not shown. Scale bar is $200 \mu \mathrm{m}$. (JPEG $856 \mathrm{~kb}$ )

Additional file 4: Figure S4. Tamoxifen was administered at E8.5 to CreER;Lhx2 ${ }^{10 \times / 10 x} ;$ Pax $6^{10 x / 10 x}$ animals and the embryos were harvested at E12.5. sFrp2 expression is not detectable in the Dbx1 expressing antihem of the double mutant embryos. (JPEG $913 \mathrm{~kb}$ )
\end{abstract}

\section{Abbreviations}

LIM-HD: LIM homeodomain

Acknowledgements

We thank A. Pierani (Dbx1, Fgf17), C. Ragsdale (Wnt2b, Wnt3a), E. Grove

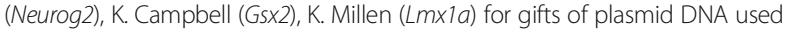
for generating RNA probes; L. D'souza and S. Sakhalkar for technical assistance; 
S. Suryavanshi and the animal house staff of the Tata Institute for Fundamental Research (TIFR) for excellent support; Edwin Monuki (Lhx $2^{\text {lox/lox }}$ ), Forbes D. Porter $\left(L h \times 2^{-1}\right)$, David Price $\left(\right.$ Pax $\left.6^{\text {lox/lox }}\right)$, and Anastassia Stoykova $\left(\right.$ Pax $\left.6^{\text {Sey/sey }}\right)$ for the kind gift of mouse lines.

\section{Funding}

This work was supported by a grant from the Department of Biotechnology, Govt. of India (PR8681) and intramural funds from TIFR-DAE (ST), by a Wellcome Trust-Department of Biotechnology India Alliance Early Career Fellowship (GG), and Sarojini Damodaran travel award from the TIFR Endowment Fund (AS). ST is a recipient of the Shanti Swarup Bhatnagar award (Council of Scientific and Industrial Research, Government of India) and the Infosys Prize for Life Sciences (Infosys Science Foundation).

None of the aforementioned funding bodies have been involved in designing the study or collection, analysis, and interpretation of data and in writing the manuscript.

\section{Availability of data and materials}

Data sharing not applicable to this article as no datasets were generated or analysed during the current study.

\section{Authors' contributions}

GG designed the study, performed the experiments and analyses in Figs. 2, 3 , and 4 and Figures S2, S3, and S4. generated the double conditional mutants; prepared the figures. AR designed the study, performed the $L h \times 2^{-/-}$, Pax $6^{\text {sey/sey }}$ and the double null experiments in Fig. 1 and Additional file 1: Figure S1, and contributed to writing the manuscript. ASS conceived the project, designed the study and helped to generate the double mutants, and contributed to writing the manuscript. ST conceived the project, designed the study, and wrote the manuscript. All authors read and approved the final manuscript.

\section{Authors information}

AR is currently at the Center for Integrative Brain Research, Seattle Children's Research Institute, Seattle, WA, USA.

ASS is currently at the Department of Stem Cell and Regenerative Biology, Harvard University, Cambridge, MA, USA.

\section{Ethics approval}

All animal protocols were approved by the Institutional Animal Ethics Committee (Tata Institute of Fundamental Research, Mumbai, India) according to regulations devised by the Committee for the Purpose of Control and Supervision of Experiments on Animals (CPCSEA), India. (Reference Approval number: TIFR/IAEC/2012-11).

\section{Consent for publication}

Not Applicable.

\section{Competing interests}

The authors declare that they have no competing interests.

\section{Publisher's Note}

Springer Nature remains neutral with regard to jurisdictional claims in published maps and institutional affiliations.

Received: 20 June 2017 Accepted: 31 October 2017

Published online: 15 November 2017

\section{References}

1. Grove EA, et al. The hem of the embryonic cerebral cortex is defined by the expression of multiple Wnt genes and is compromised in Gli3-deficient mice. Development. 1998;125:2315-25.

2. Furuta Y, Piston DW, Hogan BL. Bone morphogenetic proteins (BMPs) as regulators of dorsal forebrain development. Development. 1997;124:2203-12

3. Assimacopoulos S, Grove EA, Ragsdale CW. Identification of a Pax6dependent epidermal growth factor family signaling source at the lateral edge of the embryonic cerebral cortex. J Neurosci. 2003;23:6399-403.

4. Mangale VS, et al. Lhx2 selector activity specifies cortical identity and suppresses hippocampal organizer fate. Science. 2008;319:304-9.

5. Bulchand $\mathrm{S}$, et al. LIM-homeodomain gene Lhx2 regulates the formation of the cortical hem. Mech Dev. 2001;100:165-75.
6. Bishop KM, Goudreau G, O'Leary DD. Regulation of area identity in the mammalian neocortex by Emx2 and Pax6. Science. 2000;288:344-9.

7. Bulchand S, Subramanian L, Tole S. Dynamic spatiotemporal expression of LIM genes and cofactors in the embryonic and postnatal cerebral cortex. Dev Dyn. 2003;226:460-9.

8. Toresson H, Potter SS, Campbell K. Genetic control of dorsal-ventral identity in the telencephalon: opposing roles for Pax6 and Gsh2. Development. 2000;127:4361-71.

9. Porter FD, et al. Lhx2, a LIM homeobox gene, is required for eye, forebrain and definitive erythrocyte development. Development. 1997;124:2935-44.

10. Hill RE, et al. Mouse small eye results from mutations in a paired-like homeobox-containing gene. Nature. 1991;354:522-5.

11. Grindley JC, Davidson DR, Hill RE. The role of Pax-6 in eye and nasal development. Development. 1995;121:1433-42.

12. Stoykova A, et al. Forebrain patterning defects in small eye mutant mice. Development. 1996;122:3453-65.

13. Yun K, Potter S, Rubenstein JL. Gsh2 and Pax6 play complementary roles in dorsoventral patterning of the mammalian telencephalon. Development. 2001;128:193-205

14. Bielle F, et al. Multiple origins of Cajal-Retzius cells at the borders of the developing pallium. Nat Neurosci. 2005;8:1002-12.

15. Carney RS, et al. Differential regulation of telencephalic pallial-subpallial boundary patterning by Pax6 and Gsh2. Cereb Cortex. 2009;19:745-59.

16. Stoykova A, et al. Pax6 modulates the dorsoventral patterning of the mammalian telencephalon. J Neurosci. 2000;20:8042-50.

17. Vyas $\mathrm{A}$, et al. Paleocortex is specified in mice in which dorsal telencephalic patterning is severely disrupted. J Comp Neurol. 2003;466:545-53.

18. Simpson Tl, et al. Normal ventral telencephalic expression of Pax6 is required for normal development of thalamocortical axons in embryonic mice. Neural Dev. 2009;4:19.

\section{Submit your next manuscript to BioMed Central and we will help you at every step:}

- We accept pre-submission inquiries

- Our selector tool helps you to find the most relevant journal

- We provide round the clock customer support

- Convenient online submission

- Thorough peer review

- Inclusion in PubMed and all major indexing services

- Maximum visibility for your research

Submit your manuscript at www.biomedcentral.com/submit
) Biomed Central 